\title{
Cross-view Action Recognition over Heterogeneous Feature Spaces
}

\author{
Xinxiao $\mathrm{Wu}$ Han Wang Cuiwei Liu Yunde Jia \\ Beijing Laboratory of Intelligent Information Technology \\ School of Computer Science, Beijing Institute of Technology \\ Beijing 100081, P.R. China \\ \{wuxinxiao, wanghan, liucuiwei, jiayunde\}@bit.edu.cn
}

\begin{abstract}
In cross-view action recognition, "what you saw" in one view is different from "what you recognize" in another view. The data distribution even the feature space can change from one view to another due to the appearance and motion of actions drastically vary across different views. In this paper, we address the problem of transferring action models learned in one view (source view) to another different view (target view), where action instances from these two views are represented by heterogeneous features. A novel learning method, called Heterogeneous Transfer Discriminantanalysis of Canonical Correlations (HTDCC), is proposed to learn a discriminative common feature space for linking source and target views to transfer knowledge between them. Two projection matrices that respectively map data from source and target views into the common space are optimized via simultaneously minimizing the canonical correlations of inter-class samples and maximizing the intraclass canonical correlations. Our model is neither restricted to corresponding action instances in the two views nor restricted to the same type of feature, and can handle only a few or even no labeled samples available in the target view. To reduce the data distribution mismatch between the source and target views in the common feature space, a nonparametric criterion is included in the objective function. We additionally propose a joint weight learning method to fuse multiple source-view action classifiers for recognition in the target view. Different combination weights are assigned to different source views, with each weight presenting how contributive the corresponding source view is to the target view. The proposed method is evaluated on the IXMAS multi-view dataset and achieves promising results.
\end{abstract}

\section{Introduction}

Cross-view human action recognition has posed substantial challenges for computer vision algorithms due to the large variations from one view to another. Since the same action appears quite differently when observed from different views, action models learned from one view may degrade the performance in another view. One possible solution $[14,18,19,11]$ is building a view-independent 3D model of human body via the 3D reconstruction from multiple calibrated cameras or epipolar geometry reasoning based on point correspondences. Another strategy resort$\mathrm{s}$ to exploiting action representations that are insensitive to the changes of views, such as temporal self-similarity descriptors [4] and the view-style independent manifold representation [7]. Wu et al. [15] proposed a latent kernelized structural SVM for view-invariant action recognition where the view is modeled as a latent variable and inferred during both training and testing stage. Some other methods [17, 3] learn a separate model for each action class in each view, however, it is difficult to collect sufficient labeled samples for each view to cover all the action classes. Recently, transfer learning based methods $[2,9,20]$ have emerged to adapt the action knowledge learned on one or more views (source views) to another different view (target view) by exploring the statistical connections between them.

In this work, we propose a new transfer learning approach, namely Heterogeneous Transfer Discriminantanalysis of Canonical Correlations (HTDCC), for crossview action recognition over heterogeneous feature spaces. Our method is not restricted to action features of the same type between source view and target view, and can handle the heterogeneous action representations in the two views. Two projection matrices are learned to respectively map the source and target views to a common space, by simultaneously minimizing the canonical correlations of inter-class samples, maximizing the canonical correlations of intraclass samples, and minimizing the canonical correlation between the means of source-view and target-view samples. Instead of requiring the corresponding observation of the same action instance from source and target views, our method explores how to take advantage of label information to learn a common feature space with discrimination.

In order to adapt multiple source views to the target view, 
we additionally present a joint weight learning method to effectively combine multiple transferred source-view classifiers to generate the target-view classifiers. Since different source views perform different relations with the target view, for each source view, a specific weight is adopted to represent its closeness to the target view.

\section{Related work}

From the perspective of cross-view action recognition, some work [2, 9, 20] is closely related to our approach. Farhadi et al. [2] used maximum margin clustering to generate the splits in the source view and then transferred the split values to the target view to learn the split-based features in the target view. Their work requires feature-tofeature correspondence at the frame-level to train a classifier. Liu et al. [9] proposed a bipartite graph-based approach to learn bilingual-words from source-view and target-view vocabularies, and then transferred action models between $\mathrm{t}$ wo views via the bag-of-bilingual-words model. Zheng et al. [20] presented a transferable dictionary pair consisting of two dictionaries that correspond to the source and target views respectively, and learned the same sparse representation of each video in the pair views. These two methods rely on simultaneous observations of the same action instance from multiple views. In contrast, our method requires neither the feature-to-feature correspondence nor the video-tovideo correspondence, which significantly relaxes the requirements on the training data. Li et al. [8] proposed "virtual views" to connect action descriptors between source and target views. Each virtual view is associated with a linear transformation of the action descriptor, and the sequence of transformed descriptors can be used to compare actions from different views. Different from [8], our method can handle the cross-view action recognition when the action$\mathrm{s}$ are represented by heterogeneous features in source and target views.

From the perspective of transfer learning, our work is also related to the methods $[10,12,13,6]$ which find a "good" common feature space for source and target domains. Taylor and Cristianini [10] learned a common feature space by maximizing the correlation between the source and target training data without any label information. Shi et al. [12] proposed a Heterogeneous Spectral Mapping to discover a common feature subspace by learning two feature mapping matrices as well as the optimal projection of the data from both domains. The label information of training data from both domains is not used. Different from [10] and [12], our method does not require the sample correspondence between source and target domains. Moreover, our method utilizes the label information to discover a common feature space with more discrimination. Wang and Mahadevan [13] proposed a manifold alignment based method to learn a common feature space for all heterogeneous domains by simultaneously maximizing the intra-domain similarity and minimizing the inter-domain similarity. Their method assumes the manifold structure on the dataset. Kulis et al. [6] proposed to learn an asymmetric kernel transformation to transfer feature knowledge between source and target domains.

\section{Heterogeneous transfer discriminant- analysis of canonical correlations}

\subsection{Problem statement}

In this work, each action sample is represented by an orthogonal linear subspace of sequential image features. Denote $\mathrm{X}=\left[x_{1}, x_{2}, \ldots, x_{M}\right] \in \mathbb{R}^{D \times M}$ as the sequential image features of an action sample, where $x_{i} \in \mathbb{R}^{D}$ represents the $i$-th image feature. The orthogonal linear subspace of $\mathrm{X}$ is denoted by $\mathrm{P} \in \mathbb{R}^{D \times m}$ s.t. $\mathrm{XX}^{T}=\mathrm{P} \Lambda \mathrm{P}^{T}$, where $\Lambda$ is the $m$ largest eigenvalues and $\mathrm{P}$ is the corresponding eigenvectors. Given a large number of labeled training samples from the source view $\left\{\left.\mathrm{X}_{i}^{s}\right|_{i=1} ^{N_{s}}\right\}$ with $\mathrm{X}_{i}^{s} \in \mathbb{R}^{D_{s} \times M_{i}}$, a limited (even no) number of labeled training samples from the target view $\left\{\left.\mathrm{X}_{i}^{t}\right|_{i=1} ^{N_{t}}\right\}$ with $\mathrm{X}_{i}^{t} \in \mathbb{R}^{D_{t} \times M_{i}}$, and some unlabeled samples from the target view $\left\{\left.\mathrm{X}_{i}^{u}\right|_{i=1} ^{N_{u}}\right\}$ with $\mathrm{X}_{i}^{u} \in$ $\mathbb{R}^{D_{t} \times M_{i}}$, where the source and target samples are represented by heterogeneous image features i.e., $D_{s} \neq D_{t}$, we aim to find a common feature space of the two views as well as two projection matrices $\mathrm{T}_{s}$ and $\mathrm{T}_{t}$ for respectively mapping the source and target views to the common space.

\subsection{Background}

Discriminant-Analysis of Canonical Correlations (DCC) [5] learns a projection matrix by maximizing canonical correlations of within-class samples and minimizing canonical correlations of between-class samples. Assume $N$ training samples are given as $\left\{\left.\mathrm{X}_{i}\right|_{i=1} ^{N}\right\}$, where $\mathrm{X}_{i}$ belongs to one action class denoted by $C_{i}$. The discriminative projection matrix $\mathrm{T}=\left[t_{1}, t_{2}, \ldots, t_{m}\right] \in \mathbb{R}^{D \times m}$ defined by $\mathrm{Y}=\mathrm{T}^{T} \mathrm{X}$, where $m \leq D$ and $\left|t_{i}\right|=1$, to make the projected samples more discriminative using canonical correlations. Orthonormal subspaces of the projected data are given by $\mathrm{YY}^{T}=\left(\mathrm{T}^{T} \mathrm{X}\right)\left(\mathrm{T}^{T} \mathrm{X}\right)^{T}=\left(\mathrm{T}^{T} \mathrm{P}\right) \Lambda\left(\mathrm{T}^{T} \mathrm{P}\right)^{T}$. The matrix $\mathrm{P}$ is normalized to $\mathrm{P}^{\prime}$ so that the columns of $\mathrm{T}^{T} \mathrm{P}^{\prime}$ are orthonormal. The similarity of two projected samples is defined as the sum of canonical correlations $\mathrm{F}_{i j}=\max _{\mathrm{Q}_{i j}, \mathrm{Q}_{j i}} \operatorname{Tr}\left(\mathrm{T}^{T} \mathrm{P}_{j}^{\prime} \mathrm{Q}_{j i} \mathrm{Q}_{i j}^{T} \mathrm{P}_{i}^{\prime T} \mathrm{~T}\right)$, where the solution of $\mathrm{Q}_{i j}$ and $\mathrm{Q}_{j i}$ is given by the SVD computation $\left(\mathrm{T}^{T} \mathrm{P}^{\prime}{ }_{i}\right)^{T}\left(\mathrm{~T}^{T} \mathrm{P}_{j}^{\prime}\right)=\mathrm{Q}_{i j} \Lambda \mathrm{Q}_{i j}^{T}$. $\mathrm{T}$ is determined to maximize the similarities of any pair of intra-class samples and minimize the similarities of any pair of inter-class samples, defined by

$$
\mathrm{T}=\arg \max _{\mathrm{T}} \frac{E_{w}(\mathrm{~T})}{E_{b}(\mathrm{~T})},
$$


where $E_{w}(\mathrm{~T})=\sum_{i=1}^{N} \sum_{k \in W_{i}} \mathrm{~F}_{i k}$ and $E_{b}(\mathrm{~T})=$ $\sum_{i=1}^{N} \sum_{l \in B_{i}} \mathrm{~F}_{i l}$. The two index sets $W_{i}=\left\{j \mid C_{j}=C_{i}\right\}$ and $B_{i}=\left\{j \mid C_{j} \neq C_{i}\right\}$, respectively, denote the intra-class and inter-class samples for a given sample of class $C_{i}$.

Transfer Discriminant-Analysis of Canonical Correlations (TDCC) [16] is the extension of DCC for handling the situation when the training and testing samples have $\mathrm{d}$ ifferent data distribution properties. In order to reduce the mismatch between data distributions of different domain$\mathrm{s}$, an effective nonparametric criterion is integrated into the discriminative function in Eqn.1, formulated as

$$
\mathrm{T}=\arg \max _{\mathrm{T}} \frac{E_{w}(\mathrm{~T})+\alpha E_{r}(\mathrm{~T})}{E_{b}(\mathrm{~T})},
$$

where $E_{r}(\mathrm{~T})$ is the canonical correlation of between-view mean samples from source and target domains and $\alpha$ is the tradeoff parameter.

\subsection{Learning on heterogeneous feature spaces}

Our goal is to extend [16] to a more general case when the training data and testing data are drawn from different views with heterogeneous features. Two projection matrices are learned to respectively map the source view and target view to a common space, where the samples from the same class are closely-related to each other, the samples from $\mathrm{d}$ ifferent classes are well-separated from each other, and the data distributions of source and target views are matched to each other.

Given the source-view training data $\left\{\mathrm{X}_{i}^{s}\right\}_{i=1}^{N_{s}}$ with the corresponding labels $\left\{C_{i}^{s}\right\}_{i=1}^{N_{s}}$ where $\mathrm{X}_{i}^{s}$ denotes the $i$-th training sample from the source view and $C_{i}^{s}$ is the action class label of $\mathrm{X}_{i}^{s}$, the source-view projection matrix $\mathrm{T}_{s}=\left[t_{s, 1}, t_{s, 2}, \ldots, t_{s, d}\right] \in \mathbb{R}^{D_{s} \times d}$ is defined by $\mathrm{Y}_{i}^{s}=$ $\mathrm{T}_{s}^{T} \mathrm{X}_{i}^{s}$. Let $\mathrm{P}_{i}^{s} \in \mathbb{R}^{D_{s} \times m}$ be the orthonormal basis matrix of the $m$-dimensional linear subspace of $\mathrm{X}_{i}^{s}$, the projected $\mathrm{P}_{i}^{s}$ is $\mathrm{T}_{s}^{T} \mathrm{P}_{i}^{s \prime}$ where $\mathrm{P}_{i}^{s \prime}$ indicates the normalization of $\mathrm{P}_{i}^{s}$. Given the labeled target-view training data $\left\{\mathrm{X}_{i}^{t}\right\}_{i=1}^{N_{t}}$ with the corresponding labels $\left\{C_{i}^{t}\right\}_{i=1}^{N_{t}}$ and the unlabeled target-view training data $\left\{\mathrm{X}_{i}^{u}\right\}_{i=1}^{N_{u}}$, the target-view projection matrix $\mathrm{T}_{t}=\left[t_{t, 1}, t_{t, 2}, \ldots, t_{t, d}\right] \in \mathbb{R}^{D_{t} \times d}$ is defined by $\mathrm{Y}_{i}^{t}=\mathrm{T}_{t}^{T} \mathrm{X}_{i}^{t}$. Let $\mathrm{P}_{i}^{t}$ be the orthonormal subspace of $\mathrm{X}_{i}^{t}$, and the projected representation of $\mathrm{P}_{i}^{t}$ is $\mathrm{T}_{t}^{T} \mathrm{P}_{i}^{t^{\prime}}$ where $\mathrm{P}_{i}^{t^{\prime}}$ indicates the normalization of $\mathrm{P}_{i}^{t}$.

The learning framework of Heterogeneous Transfer Discriminant-analysis of Canonical Correlations (HTDCC) is formulated as:

$$
\max _{\mathrm{T}_{s}, \mathrm{~T}_{t}} \frac{E_{w}\left(\mathrm{~T}_{s}, \mathrm{~T}_{t}\right)+\alpha E_{r}\left(\mathrm{~T}_{s}, \mathrm{~T}_{t}\right)}{E_{b}\left(\mathrm{~T}_{s}, \mathrm{~T}_{t}\right)} .
$$

$E_{w}\left(\mathrm{~T}_{s}, \mathrm{~T}_{t}\right)=\sum_{i=1}^{N_{s}} \sum_{j \in W_{i}^{s}} \mathrm{~F}_{i j}^{s}+\sum_{i=1}^{N_{l}} \sum_{j \in W_{i}^{t}} \mathrm{~F}_{i j}^{t}+$ $\sum_{i=1}^{N_{s}} \sum_{j \in W_{i}^{s t}} \mathrm{~F}_{i j}^{s t}+\sum_{i=1}^{N_{l}} \sum_{j \in W_{i}^{t s}} \mathrm{~F}_{i j}^{t s}$ represents the similarities of intra-class training samples from both source and target views. $E_{b}\left(\mathrm{~T}_{s}, \mathrm{~T}_{t}\right)=\sum_{i=1}^{N_{s}} \sum_{j \in B_{i}^{s}} \mathrm{~F}_{i j}^{s}+$ $\sum_{i=1}^{N_{l}} \sum_{j \in B_{i}^{t}} \mathrm{~F}_{i j}^{t}+\sum_{i=1}^{N_{s}} \sum_{j \in B_{i}^{s t}} \mathrm{~F}_{i j}^{s t}+\sum_{i=1}^{N_{l}} \sum_{j \in B_{i}^{t s}} \mathrm{~F}_{i j}^{t s}$ represents the similarities of inter-class training samples from both source and target views. $\mathrm{F}_{i j}^{s}$ represents the canonical correlation of two projected samples from the source view and $\mathrm{F}_{i j}^{t}$ represents the canonical correlation of two projected samples from the target view. Both $\mathrm{F}_{i j}^{s t}$ and $\mathrm{F}_{i j}^{t s}$ represent the canonical correlations of two projected samples of which one sample is from the source view and the other sample is from the target view. They are parameterized as follows:

$$
\begin{aligned}
\mathrm{F}_{i j}^{s} & =\max _{\mathrm{Q}_{i j}^{s}, \mathrm{Q}_{j i}^{s}} \operatorname{Tr}\left(\mathrm{T}_{s}^{T} \mathrm{P}_{j}^{s \prime} \mathrm{Q}_{j i}^{s} \mathrm{Q}_{i j}^{s}{ }^{T} \mathrm{P}_{i}^{s^{\prime} T} \mathrm{~T}_{s}\right), \\
\mathrm{F}_{i j}^{t} & =\max _{\mathrm{Q}_{i j}^{t}, \mathrm{Q}_{j i}^{t}} \operatorname{Tr}\left(\mathrm{T}_{t}^{T} \mathrm{P}_{j}^{t^{\prime}} \mathrm{Q}_{j i}^{t} \mathrm{Q}_{i j}^{t}{ }^{T} \mathrm{P}_{i}^{t^{\prime T}} \mathrm{~T}_{t}\right), \\
\mathrm{F}_{i j}^{s t} & =\max _{\mathrm{Q}_{i j}^{s t}, \mathrm{Q}_{j i}^{s t}} \operatorname{Tr}\left(\mathrm{T}_{t}^{T} \mathrm{P}_{j}^{t^{\prime}} \mathrm{Q}_{j i}^{s t} \mathrm{Q}_{i j}^{s t}{ }^{t} \mathrm{P}_{i}^{s^{\prime} T} \mathrm{~T}_{s}\right), \\
\mathrm{F}_{i j}^{t s} & =\max _{\mathrm{Q}_{i j}^{t s}, \mathrm{Q}_{j i}^{t s}} \operatorname{Tr}\left(\mathrm{T}_{s}^{T} \mathrm{P}_{j}^{s \prime} \mathrm{Q}_{j i}^{t s} \mathrm{Q}_{i j}^{t s}{ }^{t} \mathrm{P}_{i}^{t^{\prime}} \mathrm{T}_{t}\right),
\end{aligned}
$$

with the solutions:

$$
\begin{aligned}
& \left(\mathrm{T}_{s}^{T} \mathrm{P}_{i}^{s \prime}\right)^{T}\left(\mathrm{~T}_{s}^{T} \mathrm{P}_{j}^{s \prime}\right)=\mathrm{Q}_{i j}^{s} \Lambda \mathrm{Q}_{j i}^{s}{ }^{T}, \\
& \left(\mathrm{~T}_{t}^{T} \mathrm{P}_{i}^{t^{\prime}}\right)^{T}\left(\mathrm{~T}_{t}^{T} \mathrm{P}_{j}^{t^{\prime}}\right)=\mathrm{Q}_{i j}^{t} \Lambda \mathrm{Q}_{j i}^{t}{ }^{T}, \\
& \left(\mathrm{~T}_{s}^{T} \mathrm{P}_{i}^{s \prime}\right)^{T}\left(\mathrm{~T}_{t}^{T} \mathrm{P}_{j}^{t^{\prime}}\right)=\mathrm{Q}_{i j}^{s t} \Lambda \mathrm{Q}_{j i}^{s t}{ }^{T}, \\
& \left(\mathrm{~T}_{t}^{T} \mathrm{P}_{i}^{t^{\prime}}\right)^{T}\left(\mathrm{~T}_{s}^{T} \mathrm{P}_{j}^{s \prime}\right)=\mathrm{Q}_{i j}^{t s} \Lambda \mathrm{Q}_{j i}^{t s}{ }^{t},
\end{aligned}
$$

The index sets $W_{i}^{s}=\left\{j \mid C_{j}^{s}=C_{i}^{s}\right\}$ and $B_{i}^{s}=\left\{j \mid C_{j}^{s} \neq\right.$ $\left.C_{i}^{s}\right\}$ respectively indicate the intra-class and inter-class data from the source view for a given source-view sample of class $C_{i}^{s} . W_{i}^{t}=\left\{j \mid C_{j}^{t}=C_{i}^{t}\right\}$ and $B_{i}^{t}=\left\{j \mid C_{j}^{t} \neq C_{i}^{t}\right\}$ respectively indicate the intra-class and inter-class data from the target view for a given target-view sample of class $C_{i}^{t}$. $W_{i}^{s t}=\left\{j \mid C_{j}^{t}=C_{i}^{s}\right\}$ and $B_{i}^{s t}=\left\{j \mid C_{j}^{t} \neq C_{i}^{s}\right\}$ respectively indicate the intra-class and inter-class data from the target view for a given source-view sample of class $C_{i}^{s}$. $W_{i}^{t s}=\left\{j \mid C_{j}^{s}=C_{i}^{t}\right\}$ and $B_{i}^{t s}=\left\{j \mid C_{j}^{s} \neq C_{i}^{t}\right\}$ respectively indicate the intra-class and inter-class data from source view for a given target-view sample of class $C_{i}^{t}$.

$E_{r}\left(\mathrm{~T}_{s}, \mathrm{~T}_{t}\right)=\mathrm{F}_{r}^{s t}+\mathrm{F}_{r}^{t s}$ represents the similarity between the projected source-view mean sample and the projected target-view mean sample, where $\mathrm{F}_{r}^{s t}=\max _{\mathrm{Q}_{r}^{s t}, \mathrm{Q}_{r}^{t s}} \operatorname{Tr}\left(\mathrm{T}_{t}^{T} \mathrm{P}_{r}^{t^{\prime}} \mathrm{Q}_{r}^{t s} \mathrm{Q}_{r}^{s t}{ }^{T^{T}} \mathrm{P}_{r}^{s^{\prime T}} \mathrm{~T}_{s}\right)$ and $\mathrm{F}_{r}^{t s}=\max _{\mathrm{Q}_{r}^{t s}, \mathrm{Q}_{r}^{s t}} \operatorname{Tr}\left(\mathrm{T}_{s}^{T} \mathrm{P}_{r}^{s^{\prime}} \mathrm{Q}_{r}^{s t} \mathrm{Q}_{r}^{t s}{ }^{t} \mathrm{P}_{r}^{t^{\prime T}} \mathrm{~T}_{t}\right)$ by $\left(\mathrm{T}_{s}^{T} \mathrm{P}_{r}^{s^{\prime}}\right)^{T}\left(\mathrm{~T}_{t}^{T} \mathrm{P}_{r}^{t^{\prime}}\right)=\mathrm{Q}_{r}^{s t} \Lambda \mathrm{Q}_{r}^{t s^{T}} . \mathrm{P}_{r}^{s^{\prime}}$ is the normalized orthonormal subspace of the mean of source-view training samples $\mathrm{X}_{r}^{s}=\frac{1}{N_{s}} \sum_{i=1}^{N_{s}} \mathrm{X}_{i}^{s}$, and $\mathrm{P}_{r}^{t^{\prime}}$ is the normalized orthonormal subspace of the mean of target-view training samples $\mathrm{X}_{r}^{t}=\frac{1}{N_{t}+N_{u}}\left(\sum_{i=1}^{N_{t}} \mathrm{X}_{i}^{t}+\sum_{i=1}^{N_{u}} \mathrm{X}_{i}^{u}\right)$. 
By the linear algebra transformation $A^{T} B=\mathbf{I}-(A-$ $B)^{T}(A-B) / 2$, we can rewrite the objective function in Eqn. 3 as

$$
\max _{\mathrm{T}_{s}, \mathrm{~T}_{t}} \frac{\operatorname{Tr}\left(\left[\begin{array}{c}
\mathrm{T}_{s} \\
\mathrm{~T}_{t}
\end{array}\right]^{T}\left[\begin{array}{cc}
\mathrm{S}_{b}^{s} & \mathrm{~S}_{b}^{t s} \\
\mathrm{~S}_{b}^{s t} & \mathrm{~S}_{b}^{t}
\end{array}\right]\left[\begin{array}{c}
\mathrm{T}_{s} \\
\mathrm{~T}_{t}
\end{array}\right]\right)}{\operatorname{Tr}\left(\left[\begin{array}{c}
\mathrm{T}_{s} \\
\mathrm{~T}_{t}
\end{array}\right]^{T}\left[\begin{array}{cc}
\mathrm{S}_{w}^{s} & \mathrm{~S}_{w}^{t s}+\alpha \mathrm{S}_{r}^{t s} \\
\mathrm{~S}_{w}^{s t}+\alpha \mathrm{S}_{r}^{s t} & \mathrm{~S}_{w}^{t}
\end{array}\right]\left[\begin{array}{c}
\mathrm{T}_{s} \\
\mathrm{~T}_{t}
\end{array}\right]\right)},
$$

where

$$
\begin{aligned}
\mathrm{S}_{b}^{s} & =\sum_{i=1}^{N_{s}} \sum_{j \in B_{i}^{s}}\left(\mathrm{P}_{j}^{s \prime} \mathrm{Q}_{j i}^{s}-\mathrm{P}_{i}^{s \prime} \mathrm{Q}_{i j}^{s}\right)\left(\mathrm{P}_{j}^{s \prime} \mathrm{Q}_{j i}^{s}-\mathrm{P}_{i}^{s \prime} \mathrm{Q}_{i j}^{s}\right)^{T}, \\
\mathrm{~S}_{b}^{t} & =\sum_{i=1}^{N_{t}} \sum_{j \in B_{i}^{t}}\left(\mathrm{P}_{j}^{t^{\prime}} \mathrm{Q}_{j i}^{t}-\mathrm{P}_{i}^{t^{\prime}} \mathrm{Q}_{i j}^{t}\right)\left(\mathrm{P}_{j}^{t^{\prime}} \mathrm{Q}_{j i}^{t}-\mathrm{P}_{i}^{t^{\prime}} \mathrm{Q}_{i j}^{t}\right)^{T}, \\
\mathrm{~S}_{b}^{t s}= & \sum_{i=1}^{N_{t}} \sum_{j \in B_{i}^{t s}}\left(\mathrm{P}_{j}^{s \prime} \mathrm{Q}_{j i}^{t s}-\mathrm{P}_{i}^{t^{\prime}} \mathrm{Q}_{i j}^{t s}\right)\left(\mathrm{P}_{j}^{s \prime} \mathrm{Q}_{j i}^{t s}-\mathrm{P}_{i}^{t^{\prime}} \mathrm{Q}_{i j}^{t s}\right)^{T}, \\
\mathrm{~S}_{b}^{s t}= & \sum_{i=1}^{N_{s}} \sum_{j \in B_{i}^{s t}}\left(\mathrm{P}_{j}^{t^{\prime}} \mathrm{Q}_{j i}^{s t}-\mathrm{P}_{i}^{s \prime} \mathrm{Q}_{i j}^{s t}\right)\left(\mathrm{P}_{j}^{t^{\prime}} \mathrm{Q}_{j i}^{s t}-\mathrm{P}_{i}^{s \prime} \mathrm{Q}_{i j}^{s t}\right)^{T}, \\
\mathrm{~S}_{w}^{s}= & \sum_{i=1}^{N_{s}} \sum_{j \in W_{i}^{s}}\left(\mathrm{P}_{j}^{s \prime} \mathrm{Q}_{j i}^{s}-\mathrm{P}_{i}^{s \prime} \mathrm{Q}_{i j}^{s}\right)\left(\mathrm{P}_{j}^{s \prime} \mathrm{Q}_{j i}^{s}-\mathrm{P}_{i}^{s \prime} \mathrm{Q}_{i j}^{s}\right)^{T}, \\
\mathrm{~S}_{w}^{t}= & \sum_{i=1}^{N_{t}} \sum_{j \in W_{i}^{t}}\left(\mathrm{P}_{j}^{t^{\prime}} \mathrm{Q}_{j i}^{t}-\mathrm{P}_{i}^{t^{\prime}} \mathrm{Q}_{i j}^{t}\right)\left(\mathrm{P}_{j}^{t^{\prime}} \mathrm{Q}_{j i}^{t}-\mathrm{P}_{i}^{t^{\prime}} \mathrm{Q}_{i j}^{t}\right)^{T}, \\
\mathrm{~S}_{w}^{t s}= & \sum_{i=1}^{N_{t}} \sum_{j \in W_{i}^{t s}}\left(\mathrm{P}_{j}^{s \prime} \mathrm{Q}_{j i}^{t s}-\mathrm{P}_{i}^{t^{\prime}} \mathrm{Q}_{i j}^{t s}\right)\left(\mathrm{P}_{j}^{s \prime} \mathrm{Q}_{j i}^{t s}-\mathrm{P}_{i}^{t^{\prime}} \mathrm{Q}_{i j}^{t s}\right)^{T}, \\
S_{w}^{s t}= & \sum_{i=1}^{N_{s}} \sum_{j \in W_{i}^{s t}}\left(P_{j}^{t^{\prime}} Q_{j i}^{s t}-P_{i}^{s \prime} Q_{i j}^{s t}\right)\left(P_{j}^{t^{\prime}} Q_{j i}^{s t}-P_{i}^{s \prime} Q_{i j}^{s t}\right)^{T}, \\
S_{r}^{t s}= & \left(P_{r}^{s \prime} Q_{r}^{t s}-P_{r}^{t^{\prime}} Q_{r}^{t s}\right)\left(P_{r}^{s \prime} Q_{r}^{t s}-P_{r}^{t^{\prime}} Q_{r}^{t s}\right)^{T}, \\
S_{r}^{s t}= & \left(P_{r}^{t^{\prime}} Q_{r}^{s t}-P_{r}^{s \prime} Q_{r}^{s t}\right)\left(P_{r}^{t^{\prime}} Q_{r}^{s t}-P_{r}^{s \prime} Q_{r}^{s t}\right)^{T} .
\end{aligned}
$$

Finally, by the eigen-decomposition

$$
\left[\begin{array}{cc}
\mathrm{S}_{b}^{s} & \mathrm{~S}_{b}^{t s} \\
\mathrm{~S}_{b}^{s t} & \mathrm{~S}_{b}^{t}
\end{array}\right] t=\lambda\left[\begin{array}{cc}
\mathrm{S}_{w}^{s} & \mathrm{~S}_{w}^{t s}+\alpha \mathrm{S}_{r}^{t s} \\
\mathrm{~S}_{w}^{s t}+\alpha \mathrm{S}_{r}^{s t} & \mathrm{~S}_{w}^{t}
\end{array}\right] t
$$

the optimal $\mathrm{T}_{s}$ and $\mathrm{T}_{t}$ are respectively constructed by the first- $D_{s}$ rows and the last- $D_{t}$ rows of the top- $d$ eigenvectors $\left[t_{1}, t_{2}, \ldots, t_{d}\right]$.

We use an iterative optimization algorithm to find the optimized projection matrices $\mathrm{T}_{s}$ and $\mathrm{T}_{t}$. With the identity matrix $\mathrm{I}$ as the initial values of $\mathrm{T}_{s}$ and $\mathrm{T}_{t}$, the detailed algorithm of HTDCC is listed in Algorithm 1. Once the optimal $\mathrm{T}_{s}$ and $\mathrm{T}_{t}$ are found, the similarity of any two action samples is measured by first mapping them to the common space and then computing the canonical correlations between them in the common space. We apply SVM to train a classifier for each action class by using the projected labeled training data from both source and target views. For SVM, we introduce a kernel based on the similarity between any pairwise samples in the learned common space.

\section{Multiple source views combination}

Since single source view may provide partial action knowledge, it is beneficial to combine multiple source-view classifiers for improving the recognition performance in the target view. Different source views perform different correlations to the target view, and action classifiers from different source views will make different contributions to the target classifiers. Therefore, we aim to increase the chance of selecting more related source views (i.e., positive source views) and simultaneously decrease the risk of transferring less related source views (i.e., negative source views). In this paper, a joint weight learning framework is proposed to assign different combination weights to different source views based on their relevances to the target view. The target classifier is actually a combination of transferred multiple source classifiers according to the corresponding weights. Considering the limited number of labeled samples in the target view, we also utilize the unlabeled target data to learn the target-view classifier. Consequently, the weights of multiple source-view classifiers are learned by minimizing the loss function of the target-view classifier on the labeled target-view samples and the loss function based on the smoothness assumption of the unlabeled target-view samples.

Suppose we have $G$ source views and one target view, the target-view classifier for an input test sample $\mathrm{X}^{t}$ from the target view is defined by

$$
f_{t}\left(\mathrm{X}^{t}\right)=\sum_{g=1}^{G} \beta_{g} f_{s, g}\left(\mathrm{X}^{t}\right),
$$

where $\beta_{g}>0$ is the weight for the $g$-th source view, constrained by $\sum_{g=1}^{G} \beta_{g}=1$. The proposed learning framework is given by

$$
\min _{f_{t}} \Omega\left(f_{t}\right)+\lambda_{l} \Omega_{l}\left(f_{t}\right)+\lambda_{u} \Omega_{u}\left(f_{t}\right)
$$

where $\lambda_{l}>0$ and $\lambda_{u}>0$ are tradeoff parameters. The details of each term in Eqn.7 are described as follows.

$\Omega\left(f_{t}\right)=\frac{1}{2}\|\boldsymbol{\beta}\|^{2}$ controls the complexity of the target classifier $f_{t}$, where $\boldsymbol{\beta}=\left[\beta_{1}, \beta_{2}, \ldots, \beta_{G}\right]^{\mathrm{T}}$.

$\Omega_{l}\left(f_{t}\right)$ is a loss function of the target-view classifier $f_{t}$ on the labeled target-view training samples, defined as

$$
\Omega_{l}\left(f_{t}\right)=\sum_{i=1}^{N_{t}}\left\|f_{t}\left(\mathrm{X}_{i}^{t}\right)-C_{i}^{t}\right\|^{2},
$$




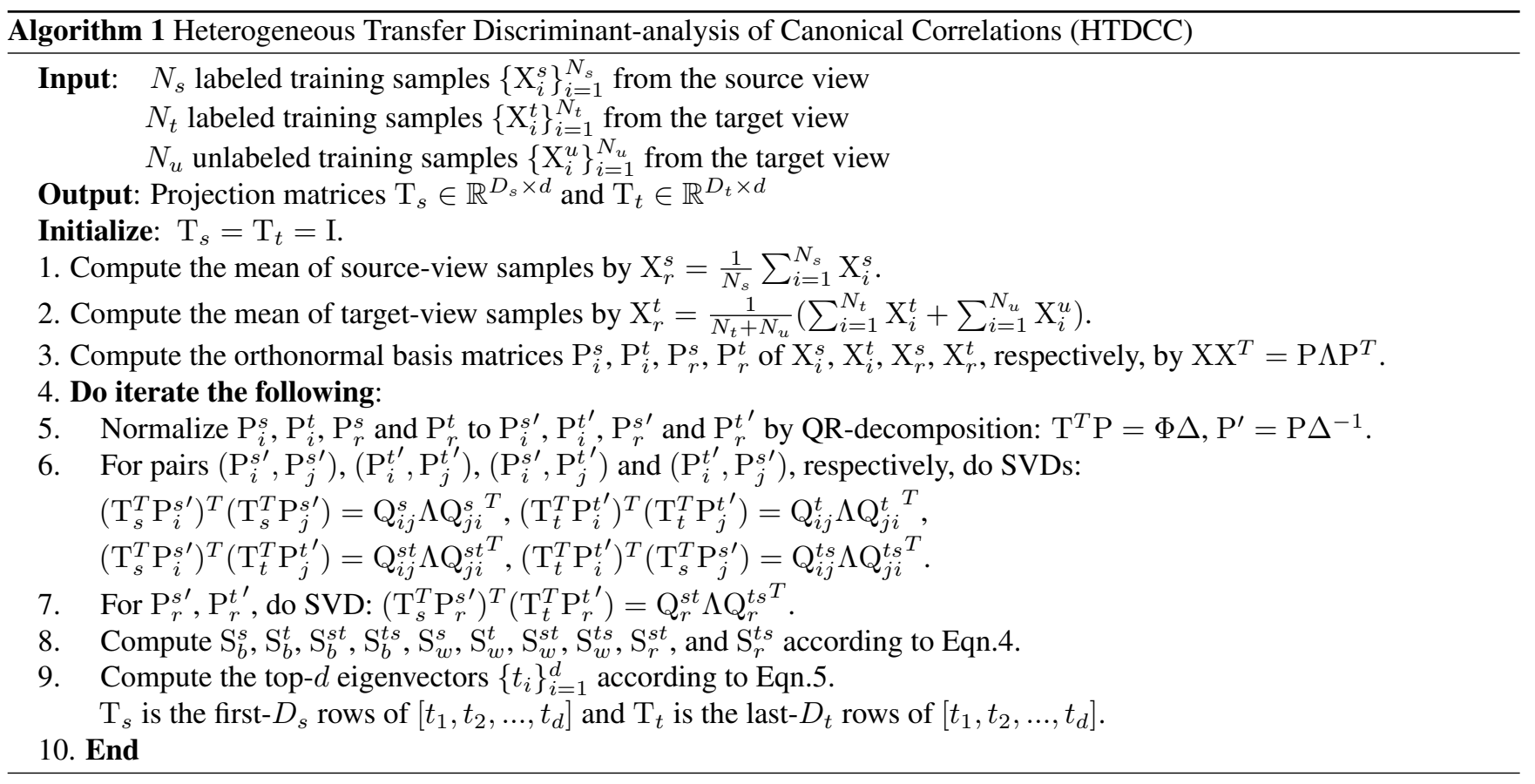

where $\mathrm{X}_{i}^{t}$ is the $i$-th labeled training sample from the target view, $C_{i}^{t}$ is the action class label of $\mathrm{X}_{i}^{t}$, and $N_{t}$ is the number of labeled target-view training samples.

$\Omega_{u}\left(f_{t}\right)$ is a group loss function based on the smoothness assumption of the unlabeled target-view data, parameterized as

$$
\Omega_{u}\left(f_{t}\right)=\sum_{g=1}^{G} \beta_{g} \sum_{k=1, k \neq g}^{G} \sum_{i=1}^{N_{u}}\left\|f_{s}^{g}\left(\mathrm{X}_{i}^{u}\right)-f_{s}^{k}\left(\mathrm{X}_{i}^{u}\right)\right\|^{2},
$$

where $\mathrm{X}_{i}^{u}$ represents the $i$-th unlabeled target-view training sample and $f_{s}^{k}$ indicates the $k$-th source-view classifier. This loss function guarantees that for each unlabeled target sample $\mathrm{X}_{i}^{u}$, its decision values of different source view classifiers should be similar to each other.

Putting all the terms together, we have the following optimization problem:

$$
\begin{aligned}
\min _{\beta} & \frac{1}{2}\|\beta\|^{2}+\sum_{i=1}^{N_{t}}\left\|f_{t}\left(\mathrm{X}_{i}^{t}\right)-C_{i}^{t}\right\|^{2} \\
& +\sum_{g=1}^{G} \beta_{g} \sum_{k=1, k \neq g}^{G} \sum_{i=1}^{N_{u}}\left\|f_{s}^{g}\left(\mathrm{X}_{i}^{u}\right)-f_{s}^{k}\left(\mathrm{X}_{i}^{u}\right)\right\|^{2} \\
\text { s.t. } & \sum_{g=1}^{G} \beta_{g}=1, \beta_{g}>0, \forall g .
\end{aligned}
$$

The optimization problem of Eqn.10 can be solved by a standard Quadratic Programming.

\section{Experiments}

\subsection{Dataset}

We evaluate the performance of our method on the IXMAS multi-view dataset [14] which consists of 11 complete action classes. Each action is executed three times by 12 subjects and recorded by 5 cameras observing the subjects from very different perspectives with the frame rate of $23 \mathrm{fps}$ and the frame size of $390 \times 291$ pixels. The body position and orientation are freely decided by different subjects.

An action video is represented by sequential images/descriptors. We extract two heterogeneous representations: sequential optical flows and sequential silhouettes, to respectively describe source-view actions and target-view actions. A silhouette descriptor is extracted from the body region and fixed to the size of $40 \times 80=3200$. An optical flow descriptor is constructed by the concatenation of four flow components with the size of $40 \times 80 \times 4=12800$. The dimension of the linear subspace for either silhouette image set or optical flow descriptor set is around 10.

\subsection{Pairwise cross-view recognition}

In this experiment, we take one view as the source view and take another different view as the target view. The optical flow feature is adopted in the source view and the silhouette feature is used in the target view. To verify the effectiveness of Heterogeneous Transfer Discriminantanalysis of Canonical Correlations (HTDCC) across pairwise views, we look into the recognition performances of all possible pairwise combinations. The leave-one-subject- 
out cross validation strategy (i.e., 12 -fold cross validation) is employed. Specifically, for each time, we use videos of one subject from the target view for testing, and use the remaining videos (i.e., videos of the rest 11 subjects) from the target view as well as all the videos from the source view as training data. For the training data, only a small number of samples from the target view and all the samples from the source view are labeled.

We compare HTDCC with the baseline method, called Heterogeneous Discriminant-analysis of Canonical Correlations (HDCC), which excludes the minimization of data distribution mismatch between source and target views in the objective function, i.e. $\alpha=0$ in Eqn.3. For these two methods, SVM is employed for classification and the regularization parameter is set to $C=1$ by choosing from $\{1,10,100,1000\}$ on the best performance. The canonical correlations based kernel is used in SVM.

Table 1 demonstrates the recognition results of HTDCC and HDCC with the fraction of labeled samples from the target view of 3/11. From Table 1, we observe that HTDC$\mathrm{C}$ is generally better than HDCC in terms of mean recognition accuracy for all the target views, which clearly demonstrates that HTDCC can successfully deal with the crossview recognition over heterogeneous feature spaces by minimizing the data distribution mismatch difference between source view and target view.

Our method is also compared with other state-of-the-art methods $[6,12,10,13,1]$ of transfer learning on heterogeneous feature spaces. For KCCA[10], HeMap[12] and DAMA[13], after learning the projection matrices, we apply SVM to train their final classifiers by using the projected training data from both views. For ARC-t[6], we construct the kernel matrix based on the learned asymmetric transformation metric, and then SVM is also applied to train its final classifier. For HFA[1], the two projection matrices for the source and target data are found by using the standard SVM with the hingeloss. For all methods, the regularization parameter $C$ in SVM is chosen from $\{1,10,100,1000\}$ according to the best performance and the linear kernel is employed.

As shown in Table 2, it is interesting to notice that HTD$\mathrm{CC}$ outperforms other methods, which clearly demonstrates the effectiveness of our method on cross-view action recognition on heterogeneous features. Compared with KCCA and HeMap, HTDCC is able to learn a common feature space with discriminative ability by using the label information of the target training data. HTDCC outperforms DAMA, possibly due to the lack of the strong manifold strcuture on this dataset. The explanation for the better performance of HTDCC than ARC-t may be that HTDC$\mathrm{C}$ utilizes unlabeled target-view training data and incorporates the minimization of the distribution mismatch between source and target views in the objective function.

\subsection{Multiple source views fusion}

We select one view as the target view and use the other four views as source views to exploit the benefits of combining multiple source views for target recognition. The parameters $\lambda_{l}$ and $\lambda_{u}$ are empirically set to $\lambda_{l}=\lambda_{u}=0.1$ by choosing from $\{0.1,1,10\}$ according to the testing performances. To verify the effectiveness of the combination weights of classifiers from multiple source views, we try a fusion method that uses equal combination weights $\beta_{g}=1 / G$, i.e., $\lambda_{l}=\lambda_{u}=0$ for comparison. To evaluate the contribution of the unlabeled target-view samples for learning the target classifier, we also report the results when excluding the loss function term defined on the unlabeled target-view training data in Eqn.7, i.e., $\lambda_{l}=0.1, \lambda_{u}=0$. To investigate the effect of the labeled target-view data, we also report the results when excluding the loss function of the labeled target-view training data in Eqn.7, i.e., $\lambda_{l}=0, \lambda_{u}=0.1$.

From the results shown in Table 3, it is interesting that: (1) the fusion of multiple source views achieves better results than each single source view because one single view has limited discriminative ability compared with multiple views; (2) assigning different combination weights to different source views can improve the recognition performance due to the selection of more related source-view classifiers transferred to the target-view classifier. Fig.1 shows some examples of learned weights of multiple source views. We can notice that the more related the source view is to the target view, the higher the learned combination weight becomes. For example, the "Target view 2" is more related to the third source view, and the weight of the third source view is higher than that of other source views.

We also report the recognition accuracy of each action class in Fig.2 which shows that the task of source-view classifier transfer is very hard for some actions and some views. For example, the recognition accuracies of "get up" and "pick up" are very low in Target view 5. One of the reasons might be that the majority of the body motions is occluded by the head in this view.

\section{Conclusions}

We have proposed a novel Heterogeneous Transfer Discriminant-analysis of Canonical Correlations (HTDCC) method for cross-view action recognition. Our method neither requires the same type of feature shared by different views nor limits to any corresponding action instances in different views. Two projection matrices are learned to respectively map the data from source and target views to a common space, by simultaneously minimizing the canonical correlations of inter-class samples, maximizing the intra-class canonical correlations, and reducing the data distribution mismatch between source and target views. Moreover, a joint weight learning method is presented to flexi- 
Table 1. Pairwise cross-view recognition accuracies using HDCC and HTDCC. Each row is a source view and each column is a target view. The two numbers in a tuple are the recognition accuracies of HDCC and HTDCC, respectively.

\begin{tabular}{|l|c|c|c|c|c|}
\hline & Target view1 & Target view2 & Target view3 & Target view4 & Target view5 \\
\hline \hline Source view1 & & $(43.1 \%, 47.2 \%)$ & $(42.4 \%, 41.0 \%)$ & $(50.7 \%, 61.8 \%)$ & $(26.4 \%, 32.6 \%)$ \\
Source view2 & $(42.4 \%, 44.4 \%)$ & & $(43.8 \%, 44.4 \%)$ & $(58.3 \%, 57.6 \%)$ & $(36.1 \%, 35.4 \%)$ \\
Source view3 & $(39.6 \%, 45.8 \%)$ & $(45.8 \%, 48.6 \%)$ & & $(55.6 \%, 54.2 \%)$ & $(29.2 \%, 37.5 \%)$ \\
Source view4 & $(45.1 \%, 43.8 \%)$ & $(43.1 \%, 41.7 \%)$ & $(43.8 \%, 43.1 \%)$ & & $(34.0 \%, 31.3 \%)$ \\
Source view5 & $(40.3 \%, 41.0 \%)$ & $(40.3 \%, 45.1 \%)$ & $(37.5 \%, 41.0 \%)$ & $(53.5 \%, 53.5 \%)$ & \\
\hline \hline Average & $(41.8 \%, 43.8 \%)$ & $(43.1 \%, 45.7 \%)$ & $(41.9 \%, 42.4 \%)$ & $(54.1 \%, 56.8 \%)$ & $(31.4 \%, 34.2 \%)$ \\
\hline
\end{tabular}

Table 2. Comparison of different heterogeneous transfer learning methods on the mean recognition accuracy for each target view.

\begin{tabular}{|l|c|c|c|c|c|c|}
\hline Methods & Target view1 & Target view2 & Target view3 & Target view4 & Target view5 & Average \\
\hline \hline KCCA [10] & $32.6 \%$ & $42.9 \%$ & $26.9 \%$ & $37.0 \%$ & $23.6 \%$ & $32.6 \%$ \\
HeMap [12] & $33.7 \%$ & $39.9 \%$ & $29.2 \%$ & $34.7 \%$ & $22.9 \%$ & $32.1 \%$ \\
DAMA [13] & $33.2 \%$ & $34.4 \%$ & $28.1 \%$ & $31.6 \%$ & $13.4 \%$ & $28.1 \%$ \\
ARC-t [6] & $29.7 \%$ & $33.2 \%$ & $32.8 \%$ & $33.5 \%$ & $15.6 \%$ & $28.9 \%$ \\
HFA [1] & $26.6 \%$ & $33.0 \%$ & $30.7 \%$ & $31.8 \%$ & $13.4 \%$ & $27.1 \%$ \\
HTDCC & $43.8 \%$ & $45.7 \%$ & $42.4 \%$ & $56.8 \%$ & $34.2 \%$ & $44.6 \%$ \\
\hline
\end{tabular}

bly combine multiple action classifiers from multiple source views for generating the target-view classifier. Experiments have shown the effectiveness of our method.

\section{Acknowledgments}

This work was supported in part by the Natural Science Foundations of China (NSFC) under Grant No.61203274, NSFC-Guangdong Joint Fund under Grant No. U1035004 and Specialized Research Fund for the Doctoral Program of Higher Education (SRFDP) under Grant No.20121101120029.

\section{References}

[1] L. Duan, D. Xu, and I. Tsang. Learning with augmented features for heterogeneous domain adaptation. In ICML, 2012.

[2] A. Farhadi and M. Tabrizi. Learning to recognize activities from the wrong view point. In ECCV, 2008.

[3] J.Liu and M. Shah. Learning human actions via information maximization. In $C V P R, 2008$.

[4] I. Junejo, E. Dexter, I. Laptev, and P. Perez. Viewindependent action recognition from temporal selfsimilarities. IEEE T-PAMI, 33(1):172-185, 2011.

[5] T. Kim, J. Kittler, and R. Cipolla. Discriminative learning and recognition of image set classes using canonical correlations. IEEE T-PAMI, 29(6):1005-1018, 2007.

[6] B. Kulis, K. Saenko, and T. Darrell. What you saw is not what you get: Domain adaptation using asymmetric kernel transforms. In CVPR, 2011.

[7] M. Lewandowski, D. Makris, and J. Nebel. View and styleindependent action manifolds for human activity recognition. In $E C C V, 2010$.
[8] R. Li and T. Zickler. Discriminative virtual views for crossview action recognition. In CVPR, 2012.

[9] J. Liu, M. Shahz, B. Kuipersy, and S. Savarese. Cross-view action recognition via view knowledge transfer. In $C V P R$, 2011.

[10] J. Shawe-Taylor and N. Cristianini. Kernel methods for pattern analysis. In Cambridge University Press, 2004.

[11] Y. Shen and H. Foroosh. View-invariant action recognition using fundamental ratios. In $C V P R, 2008$.

[12] X. Shi, Q. Liu, W. Fan, P. Yu, and R. Zhu. Transfer learning on heterogeneous feature spaces via spectral transformation. In ICDM, 2010.

[13] C. Wang and S. Mahadevan. Heterogeneous domain adaptation using manifold alignment. In IJCAI, 2011.

[14] D. Weinland, E. Boyer, and R. Ronfard. Action recognition from arbitrary views using 3d exemplars. In ICCV, 2007.

[15] X. Wu and Y. Jia. View-invariant action recognition using latent kernelized structural svm. In ECCV, 2012.

[16] X. Wu, C. Liu, and Y. Jia. Transfer discriminant-analysis of canonical correlations for view-transfer action recognition. In PCM, 2012.

[17] X.Wu, D. Xu, L. Duan, and J. Luo. Action recognition using context and appearance distribution features. In CVPR, 2011.

[18] P. Yan, S. Khan, and M. Sha. Learning 4d action feature models for arbitrary view action recognition. In CVPR, 2008.

[19] A. Yilmaz and M. Shah. Recognizing human actions in videos acquired by uncalibrated moving cameras. In $I C C V$, 2005.

[20] J. Zheng, Z.Jiang, P. Philips, and R. Chellappa. Cross-view action recognition via a transferable dictionary pair. In $B$ MVC, 2012. 
Table 3. Comparison of different multiple source views fusion methods on the recognition accuracy for each target view.

\begin{tabular}{|l|c|c|c|c|c|c|}
\hline Methods & Target view1 & Target view2 & Target view3 & Target view4 & Target view5 & Average \\
\hline \hline$\lambda_{l}=\lambda_{u}=0$ & $49.3 \%$ & $50.7 \%$ & $46.5 \%$ & $60.4 \%$ & $32.6 \%$ & $47.9 \%$ \\
$\lambda_{u}=0$ & $50.0 \%$ & $50.0 \%$ & $46.5 \%$ & $63.2 \%$ & $33.3 \%$ & $48.6 \%$ \\
$\lambda_{l}=0$ & $56.9 \%$ & $56.2 \%$ & $56.2 \%$ & $68.0 \%$ & $40.3 \%$ & $55.5 \%$ \\
Our method & $57.6 \%$ & $57.6 \%$ & $56.9 \%$ & $68.8 \%$ & $40.3 \%$ & $56.3 \%$ \\
\hline
\end{tabular}
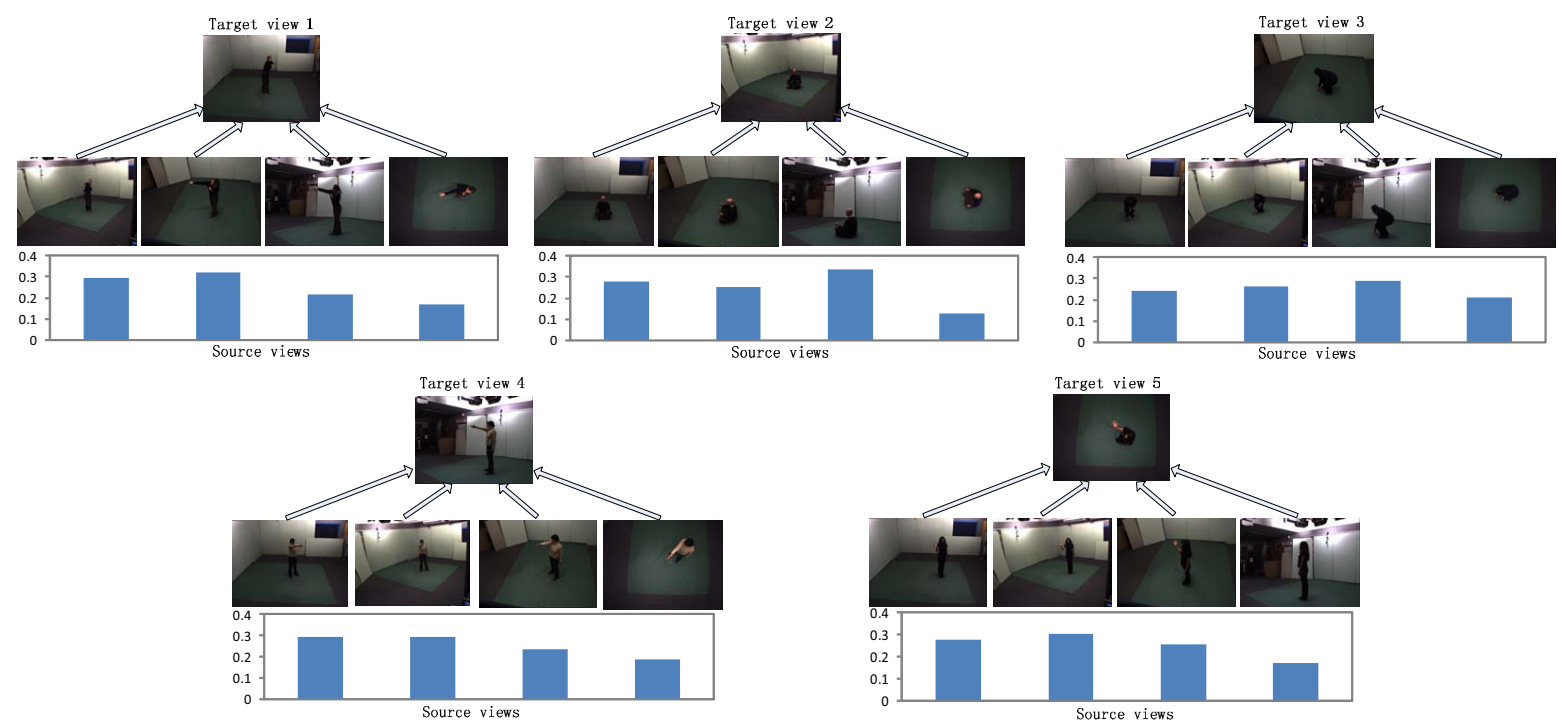

Figure 1. Examples of the learned combination weights of multiple source views. For each target view, its classifiers are constructed by the combination of transferred four source views based on the weights shown by vertical axis of histograms.

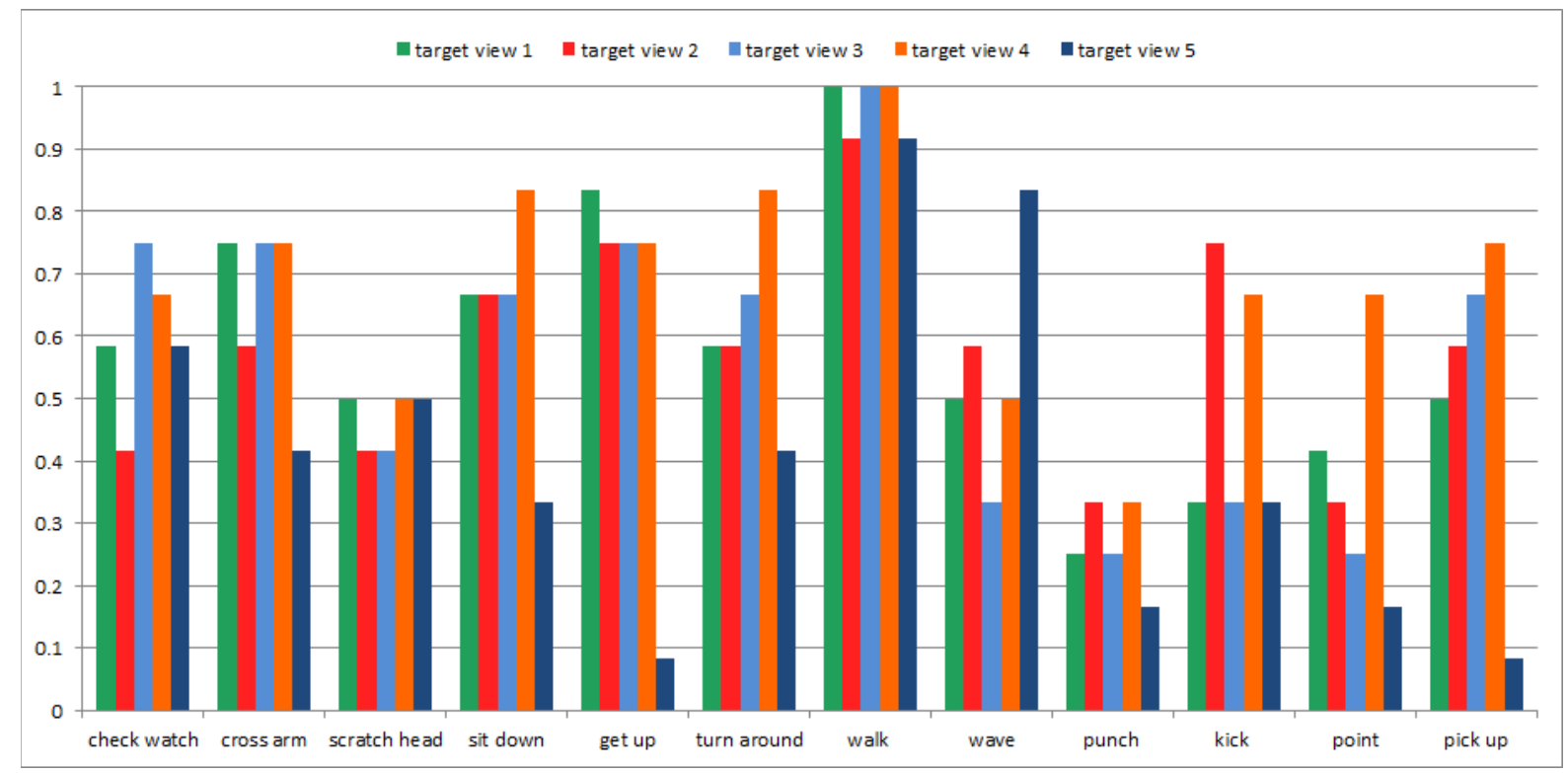

Figure 2. Recognition performance of multiple source views fusion on each action class. 